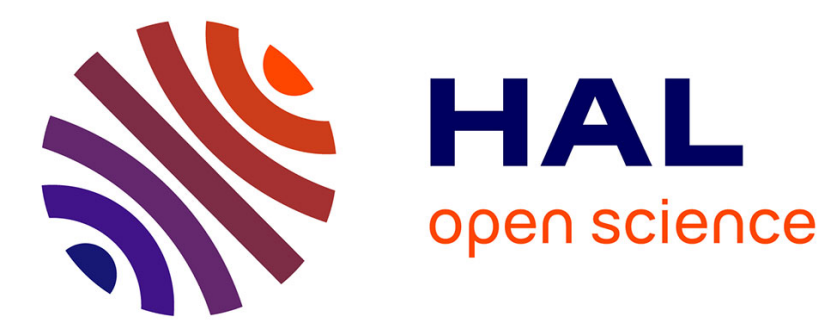

\title{
Thermoelectric properties of Cu-Sb system compounds from density functional theory calculations
}

\author{
Hailong Yang, Marie-Christine Record, P. Boulet
}

\section{To cite this version:}

Hailong Yang, Marie-Christine Record, P. Boulet. Thermoelectric properties of Cu-Sb system compounds from density functional theory calculations. Journal of the Chinese Chemical Society, 2021, 68 (6), pp.1030-1038. 10.1002/jccs.202000499 . hal-03230058

\section{HAL Id: hal-03230058 \\ https://hal.science/hal-03230058}

Submitted on 8 Feb 2022

HAL is a multi-disciplinary open access archive for the deposit and dissemination of scientific research documents, whether they are published or not. The documents may come from teaching and research institutions in France or abroad, or from public or private research centers.
L'archive ouverte pluridisciplinaire HAL, est destinée au dépôt et à la diffusion de documents scientifiques de niveau recherche, publiés ou non, émanant des établissements d'enseignement et de recherche français ou étrangers, des laboratoires publics ou privés. 


\title{
Thermoelectric properties of $\mathrm{Cu}-\mathrm{Sb}$ system compounds from DFT calculations
}

\author{
Hailong Yang, ${ }^{\mathrm{a}, \mathrm{b}}$ Marie-Christine Record ${ }^{\mathrm{b},{ }^{*}}$ and P. Boulet ${ }^{\mathrm{a}}$ \\ a MADIREL, Aix-Marseille University, CNRS, Avenue Normandie-Niemen, 13013 Marseille, France \\ b IM2NP, Aix-Marseille University, CNRS, Avenue Normandie-Niemen, 13013 Marseille, France
}

In this work the thermoelectric properties of $\mathrm{Cu}-\mathrm{Sb}$ system compounds have been investigated to understand their relation with the electronic structures. The approach used is a theoretical one that combines the density functional theory, the Boltzmann transport theory and the quantum theory of atoms in molecules. The results show that the Seebeck effect in metallic systems originates from the existence of various types of chemical bonds. However this feature is not sufficient enough to generate effective thermoelectric effect. As in semi-conducting compounds, the intrinsic driving force for thermoelectric effect remains the variation of the density of states (DOS) near the Fermi level.

Keywords: DFT; QTAIM; transport properties; chalcogenides; thermoelectrics; structure-properties relationships;

\footnotetext{
${ }^{*}$ Corresponding author: m-c.record@univ-amu.fr
} 


\section{INTRODUCTION}

As the thermoelectric (TE) properties are electronic ones, the analysis of the electronic interactions and as a consequence of bonds in materials should allow one to better understand the origin of the TE properties. For example, it has been shown in the compounds of the $\mathrm{Cu}-\mathrm{Sb}-\mathrm{S}$ system that weak chemical bondings in materials are utterly important to get a low lattice thermal conductivity. ${ }^{1}$

In the late 1960s several models of the chemical bond, namely the ionicity, ${ }^{2}$ partially ionic binding, ${ }^{3,4}$ covalent bonding ${ }^{5}$ and dielectric constants ${ }^{6}$ ones, have been developed to investigate the properties of materials. However, due to the quantum nature of the chemical bonds, working out a general model that correlates the physical properties to the bond ones is made arduous. Nonetheless, it is widely admitted that the charge density is amenable to provide information on the bonding interaction. As it turns out Bader's quantum theory of atoms in molecules (QTAIM) ${ }^{7-10}$ a topological tool applied to the electron density, is among the most advanced theories that allows for revealing bonding interaction nature in molecules and crystals. As with many other techniques, the crucial starting point is the partitioning of the whole system into subsystems. In QTAIM the molecule or the crystal is divided into the so-called atomic basins that are volumes of space containing a single atom delimited by a boundary where the flux of the electron density vanishes. From then on, the analysis of the electron density $\rho(r)$ can be achieved through that of its gradient vector field $\nabla \rho(\mathrm{r})$ and the topological analysis of the electron density boils down to the search for critical points $(\mathrm{CP})$ that are points in $\rho(\mathrm{r})$ where $\nabla \rho(\mathrm{r})$ zeroes out. The electron density of stable crystals exhibits four types of $\mathrm{CP}$ among which the bond critical point (BCP) that characterizes, together with the bond path, the bonding interaction between two atoms. The bond path is the line joining two atoms where the electron density is maximal with respect to any neighbouring path. The $\mathrm{BCP}$ is characterized by the signature $(3,-1)$, where 3 corresponds to the dimensionality and -1 is the sum of the signs of the electron density Laplacian $\nabla^{2} \rho$. Within this tool, the chemical interactions have been classified into two types, namely the shared shell interactions for which $\nabla^{2} \rho$ is negative (incoming electron density) and the closed shell ones for which $\nabla^{2} \rho$ is positive (outgoing electron density). ${ }^{11}$ Nonetheless, in many situations, in particular in semiconductors, this basic classification is insufficient and the electron density Laplacian information can be advantageously supplemented by both the local virial theorem that reads:

$$
\frac{\hbar^{2}}{4 m} \nabla^{2} \rho(r)=2 G(r)+V(r)
$$

where $\mathrm{G}(\mathrm{r})$ and $\mathrm{V}(\mathrm{r})$ are the kinetic and potential energy densities, respectively, and the total energy density $\mathrm{H}(\mathrm{r})$ relation:

$$
G(r)+V(r)=H(r)
$$

$\mathrm{G}(\mathrm{r})$ can be estimated using the approximation derived by Kirzhnits ${ }^{12,13}$ and subsequently refined by Abramov ${ }^{14}$ that relates $\rho(\mathrm{r})$ to $\mathrm{G}(\mathrm{r})$ :

$$
G(r)=\frac{3}{10}\left(3 \pi^{2}\right)^{\frac{2}{3}} \rho(r)^{\frac{5}{3}}+\frac{1}{72} \frac{[\nabla \rho(r)]^{2}}{\rho(r)}+\frac{1}{6} \nabla^{2} \rho(r)
$$

From the knowledge of $\mathrm{G}(\mathrm{r}), \mathrm{V}(\mathrm{r})$ can be determined from the virial equation (1).

According to equations (1), (2) and (3) it is possible to exploit the relation:

$$
\frac{H_{b}}{\rho_{b}}=\frac{G_{b}}{\rho_{b}}\left(1-\frac{\left|V_{b}\right|}{G_{b}}\right)
$$

where $H(r) / \rho(r)$ is the bond degree (BD) defined by Espinosa ${ }^{15}$ that stands for the total energy per electron. The bond degree corresponds to the total electronic pressure and denotes the equilibrium between the pressure exerted by an electron on the others $(G(r) / \rho(r))$ and the pressure exerted by the other electrons on this one $(V(r) / \rho(r))$. $\left|V_{b}\right| / G_{b}$ expresses the balance between the potential energy and kinetic energy densities for the formation of bonds. On the basis of the electron density Laplacian and total energy density distributions, the local energy properties at the $\mathrm{BCP}$ allows one to analyse the interatomic interactions in crystals encompassing all types of bonding from the pure closed-shell interactions to the shared-shell ones. ${ }^{1,16} G_{b} / \rho_{b}$, which is an important parameter for the bonding classification, ${ }^{11,15,17}$ is related to the evolution of $H_{b} / \rho_{b}$ vs. $\left|V_{b}\right| / G_{b}$ (eq. 4). In the present work we use the latter two indicators to investigate the bonding features in $\mathrm{Cu}$-Sb system intermediate compounds.

$\mathrm{The} \mathrm{Cu}-\mathrm{Sb}$ alloys are important for various applications, especially as lead-free solder in electronic products. The $\mathrm{Cu}$-Sb system contains six intermetallic phases including $\mathrm{Cu}_{4} \mathrm{Sb} \quad \delta$ and $\mathrm{Cu}_{2} \mathrm{Sb}(\eta$ ones for which the 
compositional homogeneity ranges are significant in some of the $\mathrm{Cu}$-Sb-based higher order systems. ${ }^{18,19}$ Since the intermediate compounds in the $\mathrm{Cu}-\mathrm{Sb}$ system are metallic conductors, their thermoelectric properties have never been investigated. The Seebeck coefficient has only been measured for $\mathrm{Cu}$-rich two-phase $\mathrm{Cu}_{2} \mathrm{Sb}$ samples. ${ }^{20}$ However the investigation of the thermoelectric properties in this system could be valuable to determine the specific influences of electronic transport on thermoelectric effect and how electronic motion generates Seebeck effect. In this work, three among the six aforementioned intermetallic phases, namely $\mathrm{Cu}_{2} \mathrm{Sb}(\mathrm{P} 4 / \mathrm{nmm}){ }^{21} \mathrm{Cu}_{3} \mathrm{Sb}$ $(\mathrm{Fm} \overline{3} \mathrm{~m})^{22}$ and $\mathrm{Cu}_{4} \mathrm{Sb}(\mathrm{P} 63 / \mathrm{mmc}){ }^{23}$ have been computationally investigated. The choice for these three compounds has been dictated by the fact that they all present a large homogeneity range of compositions and temperatures, thus allowing for the formation of large ternary solid solutions. Moreover $\mathrm{Cu}_{4} \mathrm{Sb} \quad \delta \quad$ and $\mathrm{Cu}_{2} \mathrm{Sb}(\eta \quad$ are the only stable intermediate phases at room temperature. ${ }^{18,19}$

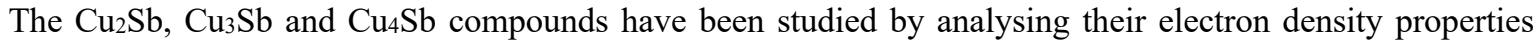
using the QTAIM approach, calculating their band structures and density of states by density functional theory (DFT) and predicting their thermoelectric properties using the Boltzmann transport theory.

\section{COMPUTATIONAL DETAILS}

The calculations have been performed at the generalized gradient approximation of the density functional theory using the Perdew-Burke-Ernzerhof (PBE) $)^{24}$ exchange-correlation functionals. The Quantum Espresso (QE) package $^{25,26}$ has been used. Scalar-relativistic projector augmented plane wave pseudopotential have been employed and the cutoffs for the plane waves and electron density have been set to 48 Ry and 480 Ry, respectively. The Monkhorst-Pack procedure has been used to generate the $k$-points mesh for the Brillouin-zone sampling. The grid adopted in our calculations corresponds to a $4 \times 4 \times 4 k$-points. The quality of the parameterization of the calculations can be assessed from the comparison between the optimized structure parameters and the experimental ones (Table 1). For $\mathrm{Cu}_{3} \mathrm{Sb}$ and $\mathrm{Cu}_{4} \mathrm{Sb}$ the calculated parameters agree nicely with the experimental ones with a relative deviation amounting to at most $2 \%$. By contrast, for $\mathrm{Cu}_{2} \mathrm{Sb}$ a much larger relative deviation is observed with $6 \%$ for a and $\mathrm{b}$ parameters and $15 \%$ for $\mathrm{c}$ parameter. These discrepancies are hardly attributed to convergence insufficiency of the technical parameters (energy cutoff and $k$-point grid) since convergence tests have been conducted. Rather, since this type of discrepancies are often observed for tetragonal Bravais lattices with too large $a$ and $b$ parameters and too small $c$ ones or the other way around, the discrepancies should be more likely due to DFT or exchange-correlation functionals weaknesses.

Table 1: Calculated structural parameters $(\AA)$ and volume $\left(\AA^{3}\right)$ of $\mathrm{Cu}-\mathrm{Sb}$ system compounds. The corresponding experimental data taken from Ref. ${ }^{27}$ are given in parenthesis.

\begin{tabular}{|l|c|c|c|l|}
\hline Structure & $\mathrm{a}$ & $\mathrm{b}$ & $\mathrm{c}$ & Volume \\
\hline $\mathrm{Cu}_{2} \mathrm{Sb}$ & $4.260(4.002)$ & $4.260(4.002)$ & $5.184(6.104)$ & $94.06(97.8)$ \\
\hline $\mathrm{Cu}_{3} \mathrm{Sb}$ & $5.934(6.000)$ & $5.934(6.000)$ & $5.934(6.000)$ & $209.0(216)$ \\
\hline $\mathrm{Cu}_{4} \mathrm{Sb}$ & $2.695(2.688)$ & $2.695(2.688)$ & $4.240(4.328)$ & $28.51(27.08)$ \\
\hline
\end{tabular}

The transport properties calculations have been performed with the BoltzTraP program. ${ }^{28}$ For this purpose the number of k-points has been extremely increased to reach between six to ten thousand k-points in the Brillouin zone.

The thermoelectric properties have been determined as follows. The DFT-calculated bands are extrapolated using a smoothed Fourier expansion. Using the constant relaxation time approximation, the electrical conductivity and Seebeck coefficient can then be calculated according the following set of equations:

$$
\sigma_{\alpha \beta}(T, \mu)=\frac{1}{\Omega} \int \sigma_{\alpha \beta}(i, k)\left(-\frac{\partial f_{\mu}(T, \epsilon)}{\partial \epsilon}\right)
$$

where $\Omega$ is the unit cell volume, $f_{\mu}(T, \epsilon)$ the Fermi-Dirac distribution, $\mu$ the electron chemical potential, $\mathrm{T}$ the temperature, $\epsilon$ the electronic energy states, and $\sigma_{\alpha \beta}(i, k)$ the electrical conductivity tensor for a given state $i$ at $k$ point $k$.

$$
\sigma_{\alpha \beta}(i, k)=e^{2} \tau_{i, k} v_{\alpha}(i, k) v_{\beta}(i, k)
$$

From the definition of the transport function:

$$
v_{\alpha \beta}(T, \mu)=\frac{1}{e T \Omega} \int \sigma_{\alpha \beta}(i, k)\left(-\frac{\partial f_{\mu}(T, \epsilon)}{\partial \epsilon}\right)
$$

the Seebeck coefficient tensor reads:

$$
S_{\gamma \eta}(T, \mu)=\frac{v_{\alpha \eta}(T, \mu)}{\sigma_{\alpha \gamma}(T, \mu)}
$$


The power factor has been calculated as the product $\mathrm{PF}=\mathrm{S}^{2} \sigma$.

The electron density has been analyzed using the program Critic $2^{29}$ based on QTAIM. The total, kinetic and potential energy densities have been determined as follows. The kinetic energy density has been calculated from the Kirzhnits expression eq. (3). From the knowledge of the electron density and its Laplacian, the potential energy density can be determined from the virial equation (1). Hence, the total energy density is obtained as the sum of the kinetic and potential ones eq. (2).

All the crystal structures presented in the following have been generated with VESTA. ${ }^{30}$

\section{RESULTS AND DISCUSSION}

\section{The $\mathrm{Cu}_{2} \mathrm{Sb}$ compound}

In the tetragonal structure of $\mathrm{Cu}_{2} \mathrm{Sb}(\mathrm{P} 4 / \mathrm{nmm})$, the copper atoms are located on the $2 \mathrm{a}(\mathrm{Cu} 2)$ and $2 \mathrm{c}(\mathrm{Cu} 1)$ sites, and the antimony atoms are located only on the $2 \mathrm{c}$ site as it can be seen in Figure 1. Along the $\mathrm{z}$ direction the crystal structure can be regarded as a "sandwich" layered structure. The central Cu2 plane is sandwiched between two atomic mono-layers, each being constituted of alternate $\mathrm{Cu} 1 \mathrm{land} \mathrm{Sb}$ atoms, corrugated due to the strong bonding between the $\mathrm{Cu} 1$ atoms and the central $\mathrm{Cu} 2$ atoms as assessed from the high absolute value of the bond degree (pink point in Figure 2). The Cu1-Sb bonds in z direction connect these "sandwich" layers. This "sandwich" layered structure exhibits anisotropy with 2-D properties; as shown in Figure 3, besides a very high electrical conductivity, the electronic transport is more efficient in the $\mathrm{x}-\mathrm{y}$ plane than along the $\mathrm{z}$ axis. Although electron transport displays some anisotropy ascribed to the distribution of $\mathrm{Cu}-\mathrm{Sb}$ bonds, the energy density features exhibit only small differences (Figure 2), leading to a more or less equivalent electronic transport in the whole cell. This statement is in agreement with the $\mathrm{Cu} 1$ and $\mathrm{Cu} 2$ atoms having the same contributions around the Fermi level as shown in the atomic PDOS (Figure 4), which are dominated by similar 3d and 3p orbitals of both Cu1 and $\mathrm{Cu} 2$ atoms. This slightly anisotropic electronic transport could be not large enough to generate electronic entropy to drive the thermoelectric effect; indeed the Seebeck effect is negligible (around $-2 \mu \mathrm{V} / \mathrm{K}$ at $300 \mathrm{~K}$ ) near the Fermi level (Figure 5), which compares well with the experimental data (around $1 \mu \mathrm{V} / \mathrm{K}$ at $300 \mathrm{~K}$ ) reported in $\mathrm{Cu}$-rich biphased samples. ${ }^{20}$ The difference in sign of the Seebeck coefficient could be related to the presence of the secondary phase in the sample. The decent value of the Seebeck coefficient closest to the Fermi level is located at $-0.18 \mathrm{Ry}$ and originates from the large contribution of $\mathrm{Cu} 1$ and $\mathrm{Cu} 2$ atoms to the DOS. This negative value is hardly achievable by doping or alloying due to the impossibility to overcome such a large potential barrier. However, $\mathrm{Cu}_{2} \mathrm{Sb}$ can be regarded as an additional constituent of great importance for the improvement of thermoelectric materials. ${ }^{31}$ Due to their layered structure, nanosized and thin films $\mathrm{Cu}_{2} \mathrm{Sb}$ are also promising as anode candidates for Na-ion batteries, showing high capacity and very high rate performance. ${ }^{32-34}$ Moreover, near the absolute zero temperature, $\mathrm{Cu}_{2} \mathrm{Sb}$ can be a superconductor. ${ }^{35}$

\section{The $\mathrm{Cu}_{3} \mathrm{Sb}$ compound}

The cubic $\mathrm{Cu}_{3} \mathrm{Sb}(\mathrm{Fm} \overline{3} \mathrm{~m})$ compound has also two positions for the $\mathrm{Cu}$ atoms, $8 \mathrm{c}$ for $\mathrm{Cu} 1$ and $4 \mathrm{~b}$ for $\mathrm{Cu} 2$. $\mathrm{The} \mathrm{Cu} 1$ atoms are bonded with four $\mathrm{Sb}$ atoms, whereas the $\mathrm{Cu} 2$ ones interact with six $\mathrm{Sb}$ atoms forming a regular octahedral configuration (Figure 6). The combined analysis of Figure 6 and Figure 7 shows that $\mathrm{Cu}$ and $\mathrm{Sb}$ atoms form two discriminative $\mathrm{Cu}-\mathrm{Sb}$ bonds; the strong $\mathrm{Cu} 1$-Sb bonding along the $<110>$ direction, so are the $\mathrm{Cu} 1-$ $\mathrm{Cu} 2$ bonds, and the relatively weak $\mathrm{Cu} 2-\mathrm{Sb}$ bonding along the three axial directions. The same distribution of chemical bonding along the $\mathrm{x}-, \mathrm{y}-$, and $\mathrm{z}$-direction implies the same electronic transport path and then the same electrical properties, in agreement with the results depicted in Figure 8. However, the different distributions of $\mathrm{Cu} 1-\mathrm{Sb}$ and $\mathrm{Cu} 2-\mathrm{Sb}$ bonds in the whole cell generate an electronic entropy which leads to a negative Seebeck coefficient slightly above the Fermi level and a corresponding peak of thermoelectric power factor (Figure 9). Indeed the $\mathrm{Cu} 2$ atoms have more prominent contribution to DOS than $\mathrm{Cu} 1$ atoms around the Fermi level (Figure 10a). This difference in DOS could be the intrinsic factor for driving thermoelectric effect. Indeed, the octahedral configuration of $\mathrm{Cu} 2$ with $\mathrm{Sb}$ atoms allows for a longer bond length, which is more ionic according to the smaller value of $\left|V_{b}\right| / G_{b}$, the charge density deformation (Figure 7), and the leading contribution of the Cu-2s orbital in the DOS (Figure 10b). By contrast, the Cu1 atoms have more obvious contribution of $3 \mathrm{~d}$ orbitals in the DOS, and are more covalently bonded to $\mathrm{Sb}$ atoms with shorter bond length (Figure 10b and Figure 7). In fact, the Seebeck coefficient is related to the variation of the DOS near the Fermi level, corresponding to the small valley around $+0.2 \mathrm{eV}$ relative to the Fermi level in Figure 10. The negative Seebeck coefficient and the peak of the thermoelectric power factor in Figure 9 are a consequence of the DOS variation of the Cu2-s orbital from zero to maximum around the Fermi level (Figure 10b), having a close relationship with the weaker $\mathrm{Cu} 2-\mathrm{Sb}$ bonds. 
In Figure 9, the peak of thermoelectric power factor almost locates around +0.015 Ry away from the Fermi level, corresponding to an approximate doping of one electron per unit cell (u.c.). This result indicates that the thermoelectric performance is more dramatically influenced by electron doping rather than hole doping. In order to further investigate the thermoelectric performance of $\mathrm{Cu}_{3} \mathrm{Sb}$, the thermoelectric power factor is calculated at various electron doping levels (Figure 11).

When increasing the carrier concentration up to $1 \times 10^{21} \mathrm{e} / \mathrm{cm}^{3}$, the thermoelectric power factor is still very low and the thermoelectric effect is negligible. When the carrier concentration reaches $5 \times 10^{21} \mathrm{e} / \mathrm{cm}^{3}$, i.e. 0.6 e/u.c., the thermoelectric power factor has a tremendous increase in the whole temperature range. With a further increase in the electronic carrier concentration, the thermoelectric performance is severely degraded. By considering that the peak of thermoelectric power factor is maximal for one electron per unit cell, the optimum concentration should be around $8.4 \times 10^{21} \mathrm{e} / \mathrm{cm}^{3}$.

\section{The $\mathrm{Cu}_{4} \mathrm{Sb}$ compound}

The structurally optimized $\mathrm{Cu}_{4} \mathrm{Sb}$ has a large number of different interatomic interactions as shown in Figure 12. This crystal displays a four-layered structure along the $\mathrm{z}$ axis (Figure 13). In the first layer constituted by $\mathrm{Sb} 1, \mathrm{Sb} 3$ and $\mathrm{Cu} 4$ atoms, the strong $\mathrm{Sb} 1-\mathrm{Sb} 3$ bonding forms linear chains in the xy plane. The Sb2 atoms located in the third layer generate four weaker $\mathrm{Cu}$-Sb bonds, among which two are forming the $\mathrm{Cu}-\mathrm{Sb}$ linear chains in the xy plane involving $\mathrm{Cu} 6$ atoms, the remaining two are involving $\mathrm{Cu} 3$ atoms of adjacent copper layers. Obviously, the electronic transport differs depending on the direction (Figure 14), the conductivity around the Fermi level being much higher in the $\mathrm{y}$ direction than in the $\mathrm{x}$ and $\mathrm{z}$ ones, with the lowest value in the $\mathrm{z}$ direction. This electrical anisotropy originates from the distribution of the various interatomic interactions.

As mentioned above, the weaker $\mathrm{Cu}-\mathrm{Sb}$ bondings solely involve the $\mathrm{Sb} 2$ atoms, being all located in the third layer. Therefore the Seebeck effect generated by these bonds cannot be extended to the whole cell and the Seebeck coefficient keeps rather small and fluctuant with the variation of chemical potential (Figure 15). In addition, the lowest electrical conductivity in the z-direction can be explained by the weak interlayer bonding; indeed only the $\mathrm{Sb} 2$ atom of the third layer is bonded with the $\mathrm{Cu} 3$ atom of the adjacent layer. By contrast, the electrical conductivity is larger in the xy plane, which can be explained by the presence of two layers containing only copper atoms, leading to a good conduction. The fact that the conduction in the $\mathrm{y}$ direction surpasses that in the $\mathrm{x}$ direction could be due to the presence of $\mathrm{Cu}-\mathrm{Sb}$ - $\mathrm{Cu}$ chains oriented in the y-direction in the layers containing both $\mathrm{Cu}$ and $\mathrm{Sb}$ atoms.

\section{CONCLUSIONS}

The QTAIM approach coupled with the calculation of the electronic band structure, density of states and thermoelectric properties has allowed one to explain the origin and the anisotropy of the transport properties. It has been shown that the Seebeck effect in metallic systems, such as in $\mathrm{Cu}-\mathrm{Sb}$ system compounds, is due to the presence of different distributions of chemical bonding in the whole cell. However a local difference of bonding distributions is not sufficient enough to generate effective thermoelectric effect.

\section{ACKNOWLEDGEMENTS}

The $\mathrm{PhD}$ thesis of Mr. H. Yang is financially supported by the China Scholarship Council (CSC). This work was granted access to the HPC resources of the Centre Informatique National de l'Enseignement Supérieur (CINES), Montpellier, France under allocation A0070806881 made by the Grand Equipement National de Calcul Intensif (GENCI). It was also granted access to the HPC resources of Aix-Marseille University financed by the project Equip@Meso (ANR-10-EQPX-29-01) of the program "Investissements d'Avenir" supervised by the Agence Nationale de la Recherche.

\section{REFERENCES}

1. H. Yang, P. Boulet, M.-C. Record, J. Solid State Chem. 2020, 286, 121266.

2. P. J. Stiles, Solid State Comm. 1972, 11, 1063-1066.

3. J. C. Phillips, Phys. Rev. 1968, 168, 905-911.

4. J. C. Phillips, J. A. Van Vechten, Phys. Rev. Lett. 1969, 22, 705-708.

5. J. C. Phillips, Phys. Rev. Lett. 1967, 19, 415-417.

6. J C. Phillips, Phys. Rev. Lett. 1968, 20, 550-553.

7. R. F. W. Bader, W. H. Henneker, P. E. Cade, J. Chem. Phys. 1967, 46, 3341-3363.

8. R. F. W. Bader, H. J. T. Preston, Int. J. Quant. Chem. 1969, 3, 327-347.

9. R. F. W. Bader, M. E. Stephens, J. Am. Chem. Soc. 1975, 97, 7391-7399. 
10. R. F. W. Bader, H. J. Essén, Chem. Phys. 1984, 80, 1943-1960.

11. C.Gatti, Z. Kristallogr. - Cryst. Mater. 2005, 220, 399-457.

12. D. A. Kirzhnits, Sov. Phys. - JETP 1957, 5, 64.

13. D. A. Kirzhnits, Field theoretical methods in many-body systems. Pergamon Press, Long Island City, New York, 1967.

14. Y. A. Abramov, Acta Crystallogr. Sect. A: Found. Crystallogr. 1997, 53, 264-272.

15. E. Espinosa, I. Alkorta, J. Elguero, J. Chem. Phys. 2002, 117, 5529-5542.

16. H. Yang, P. Boulet, M.-C. Record, Comput. Theor. Chemistry, 2020, 1178, 112784.

17. G. Gervasio, R. Bianchi, D. Marabello, Chem. Phys. Lett. 2004, 387, 481-484.

18. W. Gierlotka, D. Jendrzejczyk-Handzlik, J. Alloys Compd. 2009, 484, 172-176.

19. S. Fürtauer, H. Flandorfer, Monatsh. Chem. 2012, 143, 1275-1287.

20. W. B. Pearson, Can. J. Phys. 1964, 42, 519-525.

21. J. Nuss, M. Jansen, Z. Anorg. Allg. Chem. 2002, 628, 1152-1157.

22. W. Hofmann, Z. Metallkd. 1941, 33, 373-373.

23. M. I. Abdeeva, T. K. Rafinov, ZH. Neorgan. Khim. 1965, 10, 1206-1210.

24. J. P. Perdew, K. Burke, M. Ernzerhof, Phys. Rev. Lett. 1996, 77, 3865-3868.

25. P. Giannozzi, S. Baroni, N. Bonini, M. Calandra, R. Car, C. Cavazzoni, D. Ceresoli, G. L. Chiarotti, M. Cococcioni, I. Dabo, J. Phys.: Condens. Matter. 2009, 21, 395502.

26. P. Giannozzi, O. Andreussi, T. Brumme, O. Bunau, M. B. Nardelli, M. Calandra, C. Car, C. Cavazzoni, D. Ceresoli, M. Cococcioni, N. Colonna, I. Carnimeo, A. Dal Corso, S. de Gironcoli, P. Delugas, R. A. DiStasio Jr, A. Ferretti, A. Floris, G. Fratesi, G. Fugallo, R. Gebauer, U. Gerstmann, F. Giustino, T. Gorni, J. Jia, M. Kawamura, H.-Y. Ko, A. Kokalj, E. Küçükbenli, M. Lazzeri, M. Marsili, N. Marzari, F. Mauri, N. L. Nguyen, H.-V. Nguyen, A. Otero-de-la-Roza, L. Paulatto, S. Poncé, D. Rocca, R. Sabatini, B. Santra, M. Schlipf, A. P. Seitsonen, A. Smogunov, I. Timrov, T. Thonhauser, P. Umari, N. Vast, X. Wu, S. Baroni, J. Phys.: Condens. Matter. 2017, 29, 465901.

27. P. Villars, K. Cenzual, Pearson' s Crystal Data, Crystal Structure Database for Inorganic Compounds, Release 2010/11, ASM International, Materials Park, OH, USA, 2011.

28. G. K. H. Madsen, D. J. Singh, Comput. Phys. Comm. 2006, 175, 67-71.

29. A. Otero-de-la-Roza, E. R. Johnson, V. Luaña, Comput. Phys. Comm. 2014, 185, 1007-1018.

30. K. Momma, F. Izumi, J. Appl. Crystallogr. 2011, 44, 1272-1276.

31. J. L. Cui, L. D. Mao, D. Y. Chen, X. Qiang, X.L. Liu, W. Yang, Curr. Appl. Phys. 2009, 9, 713-716.

32. J. M. Mosby, A. L. Prieto, J. Am. Chem. Soc. 2008, 130, 10656-10661.

33. L. Baggetto, E. Allcorn, A. Manthiram, G. M. Veith, Electrochem. Comm. 2013, 27, 168-171.

34. L. Baggetto, K. J. Carroll, H. Y. Hah, C. E. Johnson, D. R. Mullins, R. R. Unocic, J. A. Johnson, Y. S. Meng, G. M. Veith, J. Phys. Chem. C2014, 118, 7856-7864.

35. K. Andres, E. Bucher, J. P. Maita, A. S. Cooper, Phys. Lett. A, 1968, 28, 67-68. 


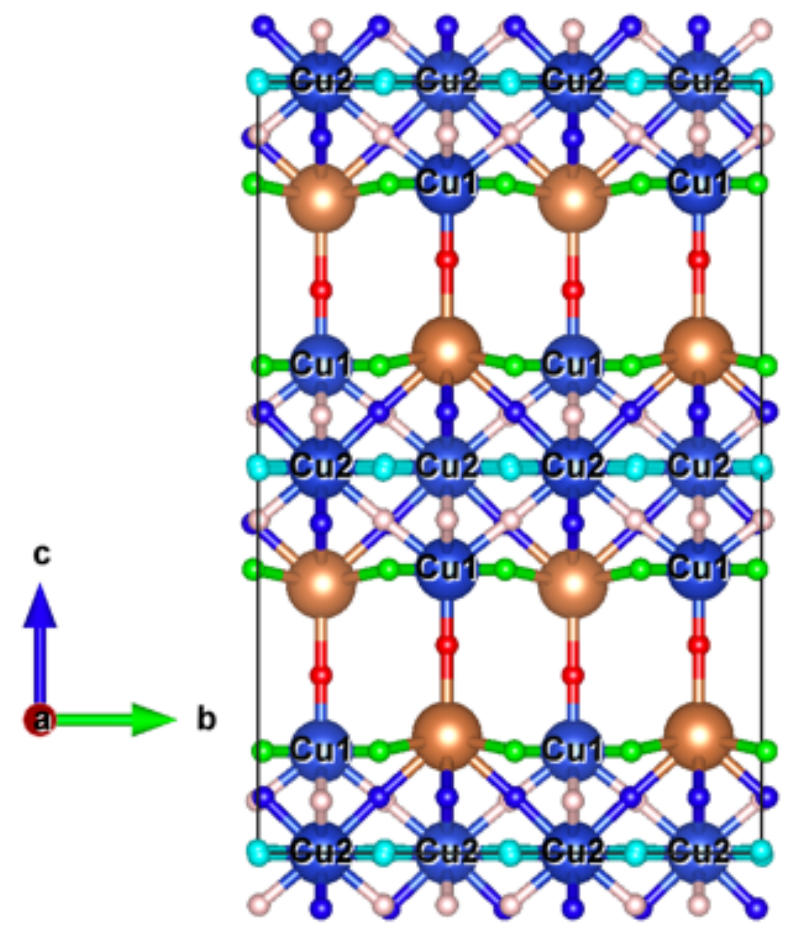

Fig. 1. Supercell of $\mathrm{Cu}_{2} \mathrm{Sb}(\mathrm{P} 4 / n m m)$. The $\mathrm{a}, \mathrm{b}$ and $\mathrm{c}$ directions correspond to the $\mathrm{x}, \mathrm{y}$ and $\mathrm{z}$ ones respectively. The small color balls are BCPs of different interactions.

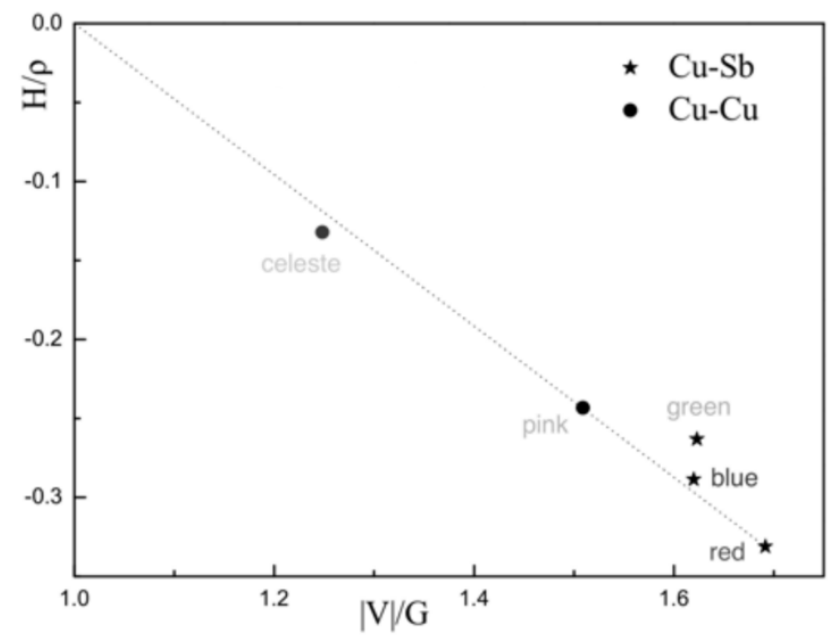

Fig. 2. $H_{b} / \rho_{b}$ vs $\left|V_{b}\right| / G_{b}$ for interactions in $\mathrm{Cu}_{2} \mathrm{Sb}$. The dash line passes through the point $(1,0)$ and that of the shortest $\mathrm{Cu}$ $\mathrm{Sb}$ bond. The color words indicate the corresponding colors of the BCP in Fig. 1. 


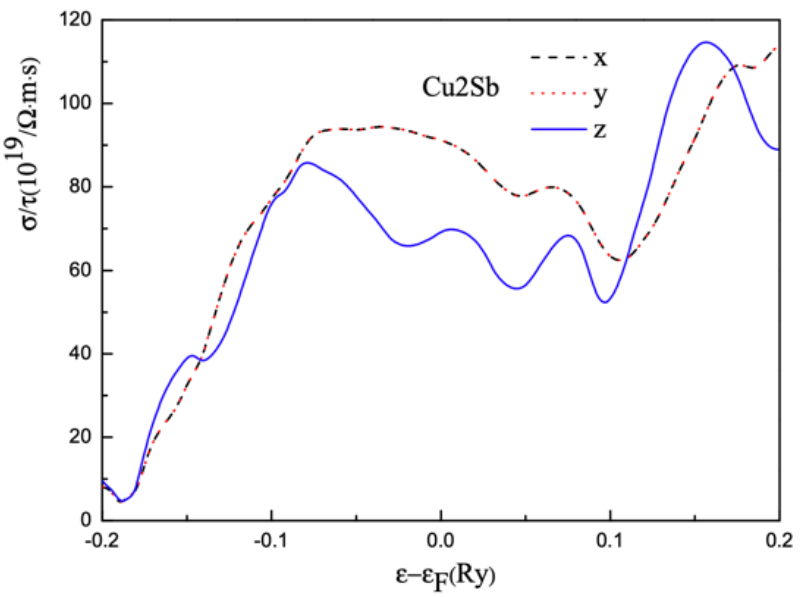

fig.3. Electrical conductivity as a function of chemical potential in different directions at $300 \mathrm{~K}$ for $\mathrm{Cu}_{2} \mathrm{Sb}$.

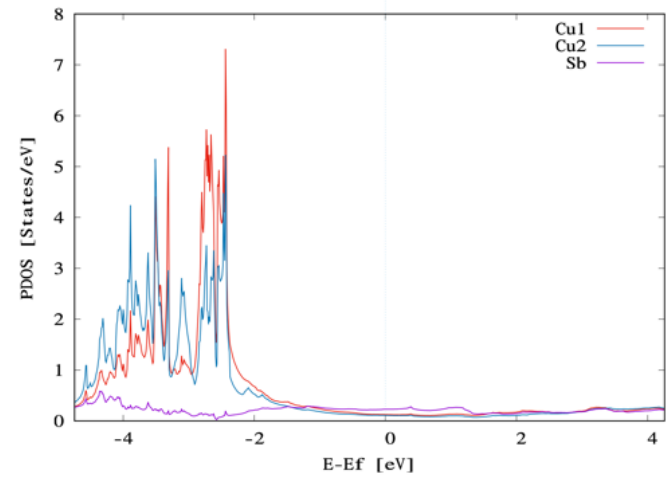

(a)

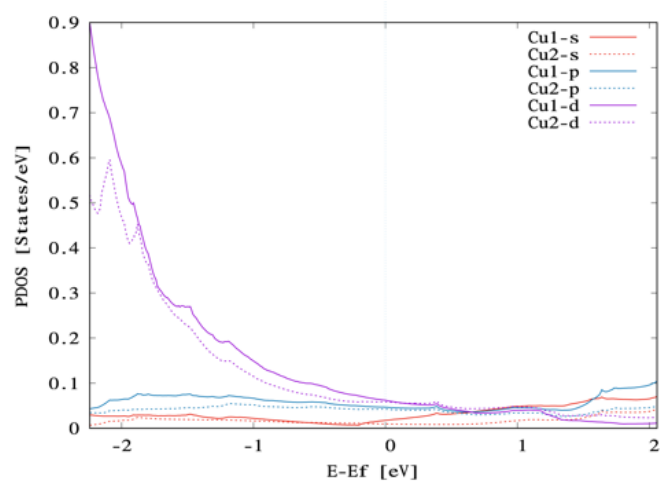

(b)

Fig. 4. PDOS in $\mathrm{Cu}_{2} \mathrm{Sb}$ : a) contributions of the atoms ; b) contributions of the atomic orbitals. 

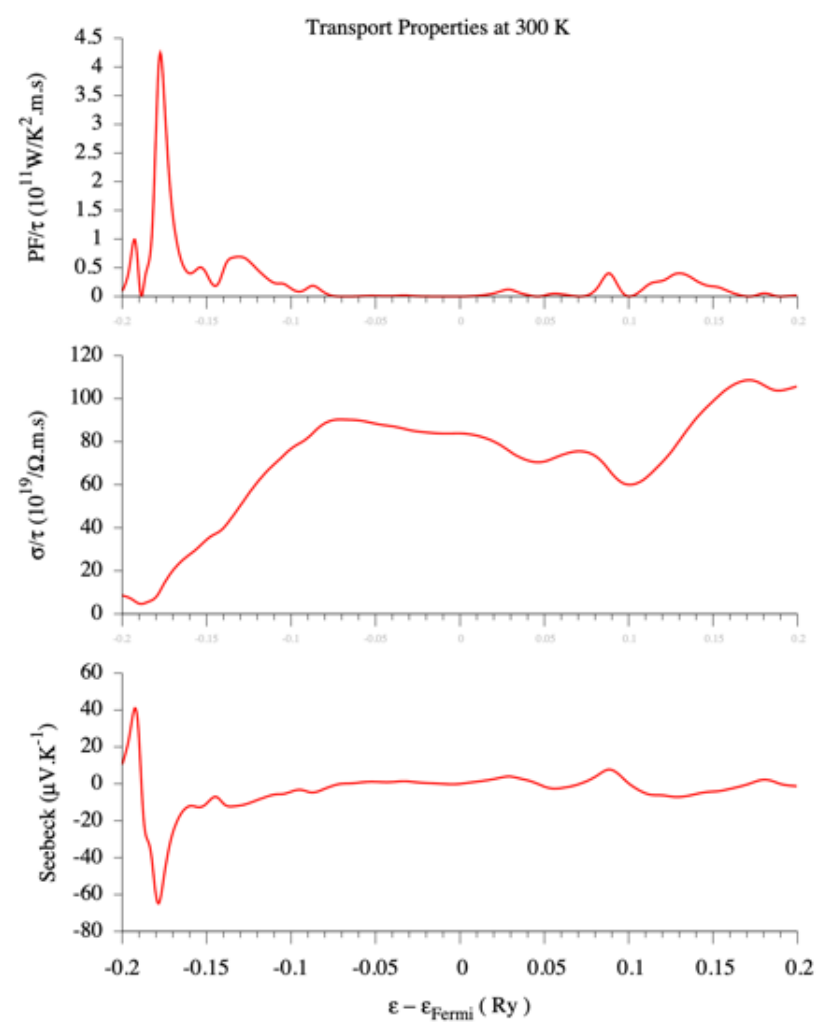

Fig. 5. Thermoelectric properties (Seebeck coefficient, electrical conductivity and power factor) as a function of the chemical potential at $300 \mathrm{~K}$ for $\mathrm{Cu}_{2} \mathrm{Sb}$.

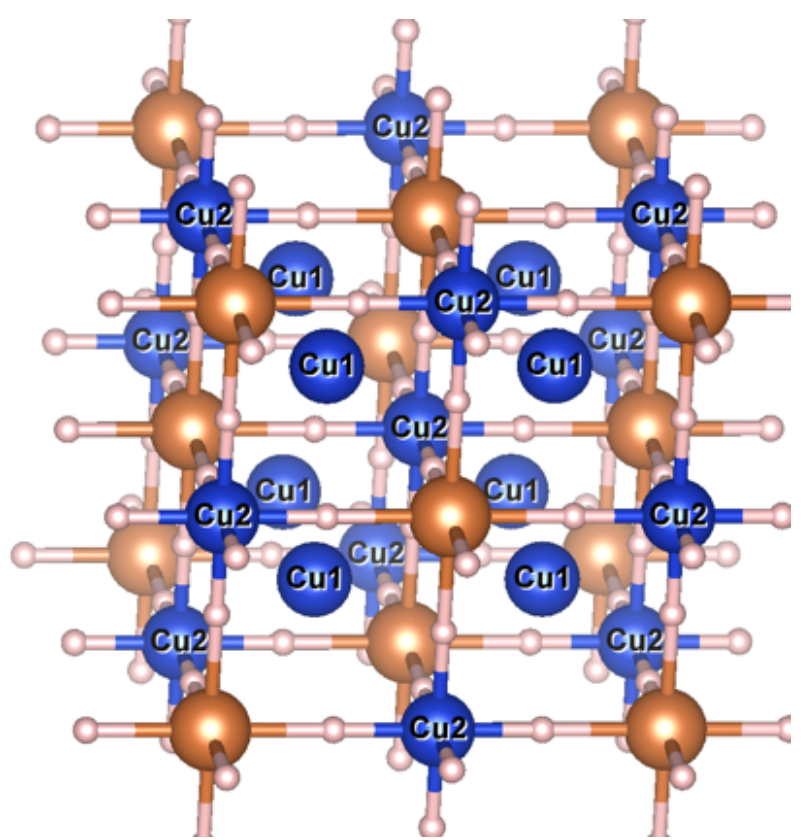

Fig. 6. Crystal structure of $\mathrm{Cu}_{3} \mathrm{Sb}(F m \overline{3} m)$. The small pink balls are BCPs of weaker $\mathrm{Cu}-\mathrm{Sb}$ bonds. 


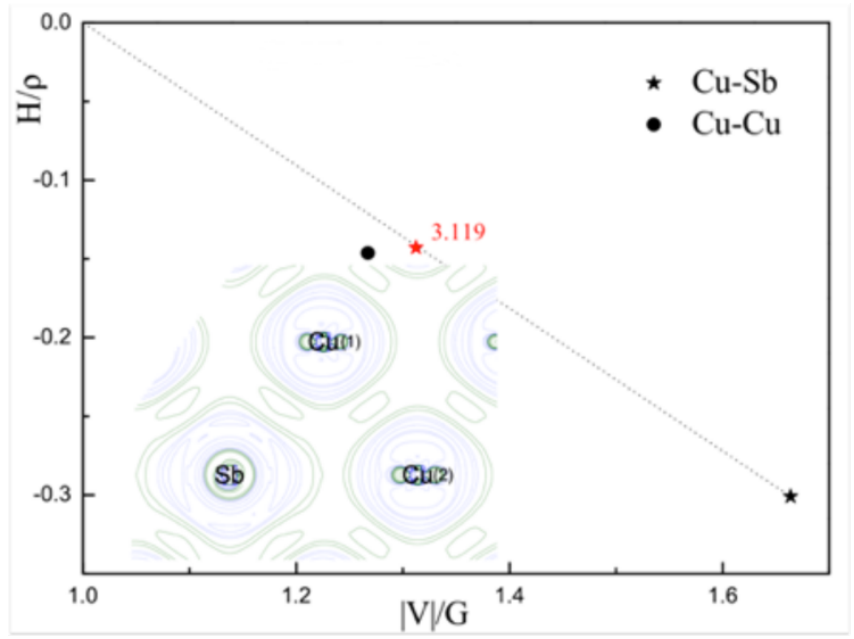

Fig. 7. $\mathrm{H}_{b} / \rho_{b}$ vs. $\mid \mathrm{V}_{\mathrm{b}} / \mathrm{G}_{\mathrm{b}}$ for interactions in $\mathrm{Cu}_{3} \mathrm{Sb}$. The dash line passes through the point $(1,0)$ and that of the shortest $\mathrm{Cu}-\mathrm{Sb}$ bond (black symbol). The insert shows the charge density deformation of $\mathrm{Cu} 1-\mathrm{Sb}$ and $\mathrm{Cu} 2-\mathrm{Sb}$. Green and blue contour curves stand for positive and negative values, respectively. The red symbol corresponds to the weaker $\mathrm{Cu} 2$-Sb bond and the associated number corresponds to its interatomic distance in $\AA$.

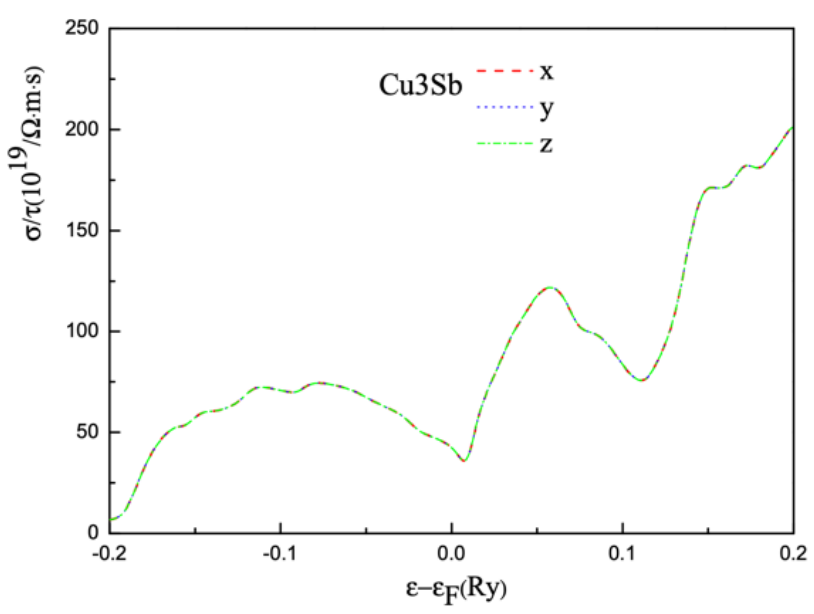

Fig. 8. Electrical conductivity as a function of chemical potential in different directions at $300 \mathrm{~K}$ for $\mathrm{Cu}_{3} \mathrm{Sb}$. 

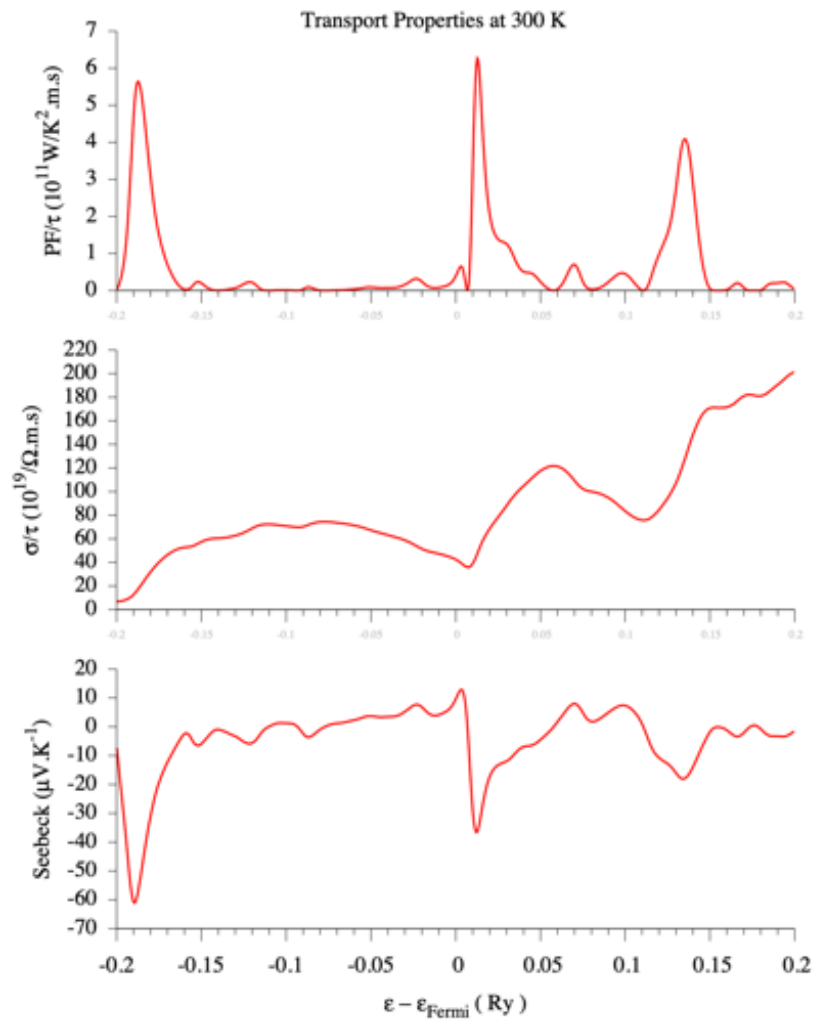

Fig. 9. Thermoelectric properties as a function of chemical potential at $300 \mathrm{~K}$ for $\mathrm{Cu}_{3} \mathrm{Sb}$.

(a)

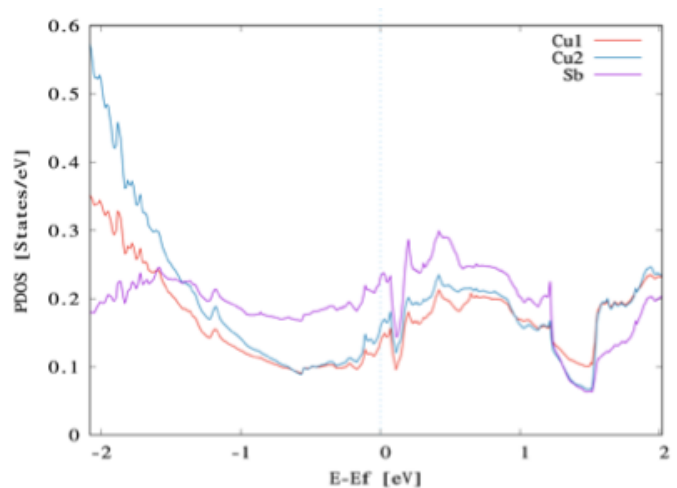




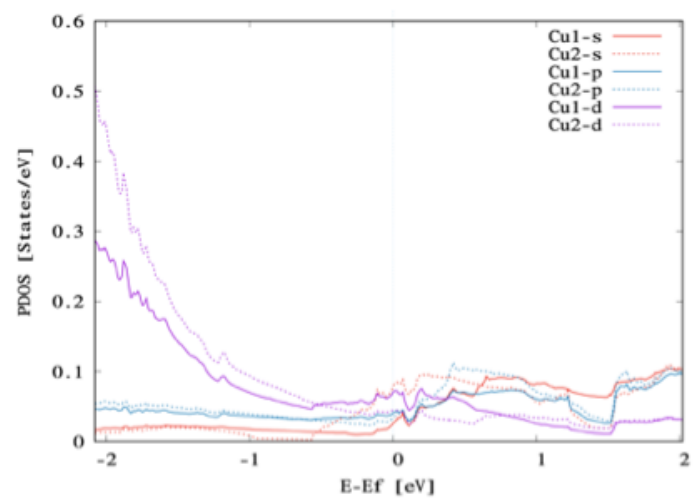

(b)

Fig. 10. PDOS in $\mathrm{Cu}_{3} \mathrm{Sb}$ : a) contributions of the atoms ; b) contributions of the atomic orbitals.

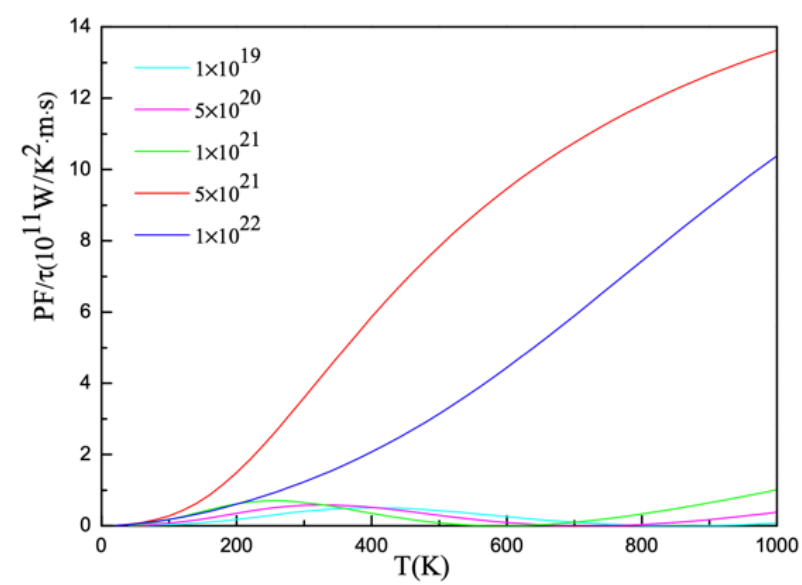

Fig. 11. Thermoelectric power factor as a function of temperature for various carrier concentrations $\left(\mathrm{e} / \mathrm{cm}^{3}\right)$ in $\mathrm{Cu}_{3} \mathrm{Sb}$.

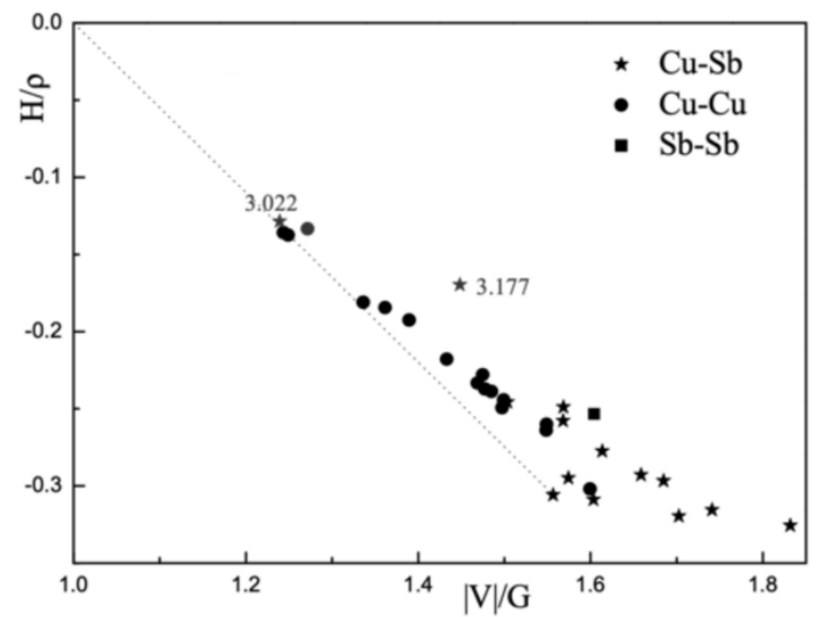


Fig. 12. $\mathrm{H}_{\mathrm{b}} / \rho_{\mathrm{b}}$ vs. $\mid \mathrm{V}_{\mathrm{b}} / \mathrm{G}_{\mathrm{b}}$ for interactions in $\mathrm{Cu}_{4} \mathrm{Sb}$. The dash line passes through the point $(1,0)$ and that of the shortest $\mathrm{Cu}-\mathrm{Sb}$ bond. The numbers correspond to interatomic distances in $\AA$.

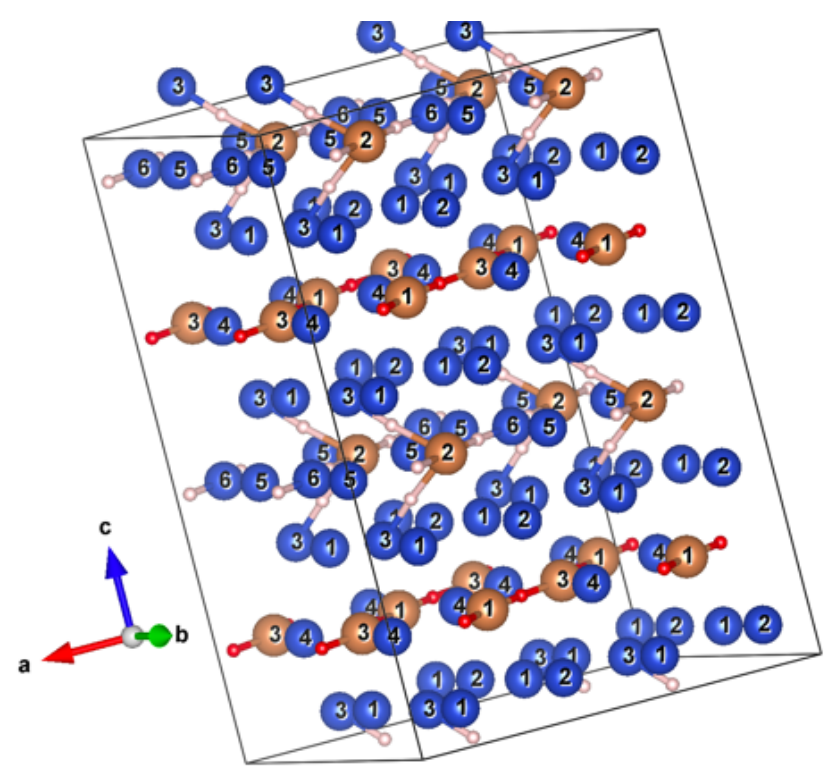

Fig. 13. Supercell of $\mathrm{Cu}_{4} \mathrm{Sb}$. The small pink balls are BCPs of weaker $\mathrm{Cu}-\mathrm{Sb}$ bonds and the red ones are BCPs of Sb-Sb bonds.

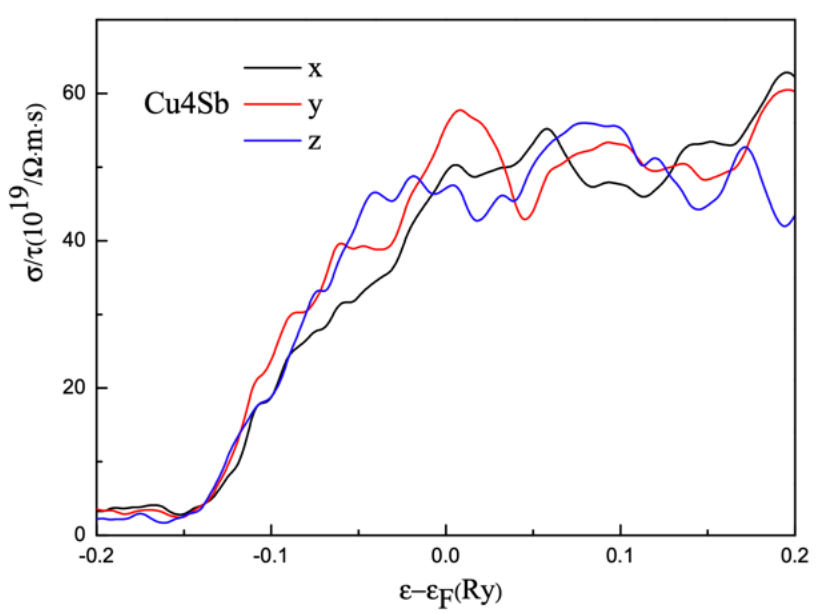


Fig. 14. Electrical conductivity as a function of chemical potential in different directions at $300 \mathrm{~K}$ for $\mathrm{Cu}_{4} \mathrm{Sb}$.
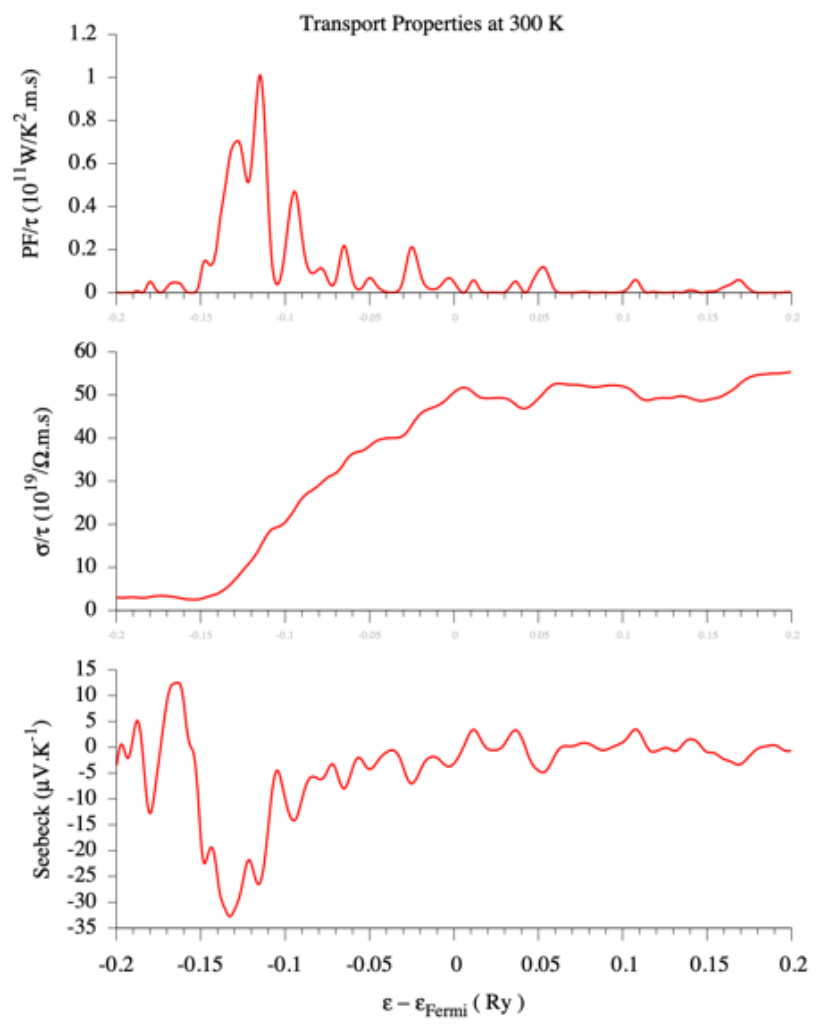

Fig. 15. Thermoelectric properties as a function of chemical potential at $300 \mathrm{~K}$ for $\mathrm{Cu}_{4} \mathrm{Sb}$. 